\title{
Estado del arte de experiencias sobre prácticas de enseñanza en educación a distancia en la Universidad Nacional de Rosario
}

\author{
Susana Copertari \\ Yanina N. Fantasía \\ (Escuela de Ciencias de la Educación) \\ (Universidad Nacional de Rosario. Argentina)
}

\section{Resumen}

El trabajo que presentamos se enmarca en un Proyecto de Investigación radicado en el Instituto de Investigaciones de la Facultad de Humanidades y Artes de la Universidad Nacional de Rosario (1HUM266/2008). La población sobre la que estamos investigando la integran las Facultades de la Universidad Nacional de Rosario con experiencias en carreras de grado con modalidad a distancia.

La Universidad Nacional de Rosario lleva un proceso lento en la introducción de propuestas de enseñanza con modalidad a distancia, es por ello que nos preguntamos en el marco del Proyecto de investigación: ¿de qué manera se están desarrollando las prácticas de enseñanza a distancia en la Universidad Nacional de Rosario -en carreras de grado- en relación al abordaje de los contenidos curriculares, estrategias metodológicas, enfoques epistemológicos, soportes tecnológicos utilizados, sistemas de evaluación, acreditación y Formación docente en el Área, que den cuenta de configuraciones didácticas inscriptas en la buena enseñanza (Litwin, 2000), para generar procesos de comprensión genuina en los estudiantes?

El proyecto está enmarcado en la lógica cualitativa, con triangulación intermetodológica utilizando el método comparativo continuo, con aportes tomados de la hermenéutica (Habermas, 1995) - crítica.

\section{Palabras clave}

Educación a Distancia - Prácticas de Enseñanza - Buena Enseñanza - Tecnología Educativa - Desempeños de Comprensión.

\section{Summary}

This job we're presenting frames itself in an Investigation Project located in the Investigation Institute of the Humanities and Arts Faculty of the National University of Rosario (1HUM266/2008). This Population we're searching is integrated by University of Rosario Faculties with experiences in grade careers with distance modality. 
The thing is National University of Rosario has had a slow process in the introduction to teaching proposals with a distance modality, and that's why we ask ourselves, into the Investigation Project frame: In which way teaching practices with distance modality are being developed in the National University of Rosario -in grade careers- as for the boarding of curricular contents, methodological strategies, epistemological focusing, technological supports used, evaluation systems, accreditation and Educational training in the area, which sink into the enrolled didactical configurations in the "good teaching" (Litwin, 2000), to generate genuine comprehension processes in the students.

The project is framed in a qualitative logical, with intermethodological triangulation, using comparative and constant method with contributions taken in the hermeneutics (Habermas, 1995) - criticism.

\section{Key words}

Distance Education - Teaching Practices - Good Teaching - Educative Technology Performances of Understanding.

\section{Introducción}

Las propuestas de enseñanza con modalidad a distancia en la Universidad Nacional de Rosario dan cuenta de un desarrollo lento y asistemático. Estos desarrollos han tenido como características el surgimiento de propuestas aisladas, que por lo general corresponden a departamentos, dentro de las carreras de grado o a proyectos de cátedra a partir de los años ' 90 .

Si bien la aparición de la cultura digital -adoptada en muchos casos por docentes universitarios- contribuyó a que se diseñaran e implementaran propuestas con dicha modalidad, aún no se han establecido en la Universidad Nacional de Rosario políticas académicas al respecto.

Es por ello que nuestra investigación tiene como objeto indagar acerca de las prácticas de enseñanza en las Facultades de dicha Universidad, donde se dictan carreras de grado a distancia, fundamentalmente en lo que hace al desarrollo de las prácticas de enseñanza. Esto es, cómo se abordan los contenidos curriculares, las estrategias metodológicas, los enfoques epistemológicos, los soportes tecnológicos, los sistemas de evaluación, acreditación y Formación docente en el Área, que den cuenta de configuraciones didácticas inscriptas en la "buena enseñanza", para generar procesos de comprensión genuina en los estudiantes.

En este trabajo, en primer lugar, explicitamos el encuadre metodológico que sustenta nuestra investigación, tomando el método comparativo continuo, ya sea para el análisis de las dos experiencias pioneras dentro de la lógica cualitativa, a un nivel exploratorio-descriptivo e interpretativo-crítico, con aportes tomados de la tradición hermenéutica (Habermas, 1995) y crítica, como así también, de otras experiencias en la modalidad que se vienen realizando en carreras de grado. 
En segundo lugar, desarrollamos algunas categorías y/o conceptos que nutren el marco teórico referencial. En tercer lugar, contextualizamos la Universidad Nacional de Rosario y presentamos la sistematización que hemos realizado sobre el estado del arte de experiencias en nuestra Universidad. Aclaramos que estamos iniciando en la investigación, una segunda etapa con el abordaje del campo empírico y que haremos lo propio, utilizando técnicas cualitativas con triangulación intermetodológica, para comparar, combinar y complementar la información obtenida a través del análisis documental, entrevistas semiestructuradas, focalizadas y abiertas en profundidad, que serán desarrolladas en otras presentaciones a medida que se vaya avanzando.

Actualmente -y conforme al cronograma de trabajo que nos hemos fijado- nos encontramos en la etapa de relevamiento del campo empírico y en la preparación de los instrumentos que se utilizarán. En esta comunicación estamos socializando sólo algunos de los resultados obtenidos a través de la búsqueda de información -para no excedernos de los límites fijados para esta presentación-, mediante materiales impresos en soporte papel (fuentes primarias y secundarias) y vía Internet, a modo de etapa inicial, para conocer el estado de la cuestión o del arte sobre el tema. De modo que nos detendremos sobre el estado actual de los conocimientos sobre el tema, principalmente sobre dos de las experiencias pioneras en la modalidad a distancia en nuestra Universidad (1).

Por último, y como cierre, dejamos planteadas algunas reflexiones -generales- a modo de conclusiones, ya que el estudio comparativo constante lo iremos profundizando sobre la información que vayamos obteniendo del campo empírico, a partir de las entrevistas y al análisis de los documentos.

\section{Encuadre Metodológico referencial de la Investigación}

Ante la complejidad del problema a investigar, nos propusimos recuperar experiencias de prácticas de enseñanza en Educación a Distancia en carreras de grado de la Universidad Nacional de Rosario. El abordaje empírico lo llevaremos a cabo en una segunda etapa que estaremos realizando a partir de Octubre de 2009, a través de un rastreo de documentos, entrevistas y proyectos institucionales. Indagaremos sobre los distintos enfoques, concepciones, líneas de investigación, propuestas y experiencias de los docentes que participan o participaron en estas experiencias. Al finalizar el trabajo investigativo (Diciembre de 2011), intentaremos realizar aportes que contribuyan a la problemática planteada, a la formación de profesores en el Área de la Educación a Distancia y a las políticas académicas de la Universidad Nacional de Rosario.

El carácter exploratorio-descriptivo e interpretativo-crítico de la problemática planteada nos ha llevado a optar por una estrategia metodológica cualitativa. La misma nos compromete a asumir el objeto de estudio desde una perspectiva compleja, hacia la comprensión de las incertidumbres (Morin, 1995). Para ello 
realizaremos una triangulación intermetodológica (Denzin, 1978), en donde se combinarán tres técnicas: la entrevista semiestructurada, las focalizadas y abiertas en profundidad y el análisis documental, para alcanzar una comprensión interpretativa y crítica de las prácticas de enseñanza en cada una de las experiencias, utilizando el método comparativo continuo (Bolsegui y Fuguet Smith, 2006).

"En este contexto, surgen propuestas emergentes para el abordaje de la investigación social bajo una óptica epistemológica que sugiere una comprensión del sentido de la acción social desde la perspectiva de los actores sociales, y de sus relaciones intersubjetivas, que reconoce su capacidad para crear significados. Se podría afirmar que la investigación cualitativa se ubica en el marco de estas propuestas emergentes. (...) En correspondencia con lo anteriormente mencionado, Guba y Lincoln (1989) y Fuguet (2000), recomiendan a la investigación naturalista como paradigma alternativo al científico o racionalista, consideran su conveniencia al estudiar los fenómenos sociales debido a sus características, entre las que se enumeran: relevancia contextual, énfasis en todo el proceso y no solamente en el control y la experimentación, su interés en el desarrollo de teorías que expliquen la información, y no en la búsqueda de datos que se correspondan con una teoría previa, la consideración del ser humano como principal instrumento de recolección de información, donde se admite la construcción de un conocimiento tácito: intuiciones, percepciones, insights, presentes en el investigador en virtud de su conocimiento y experiencia, y el presupuesto de que la investigación está sustentada por el sistema de valores que caracterizan al investigador, el informante, el paradigma que se elija, la teoría sustantiva que se relacione, la cual puede orientar tanto los métodos de recolección y análisis como las formas de interpretar los hallazgos, y la preferencia por contextos naturales donde el investigador observa los hechos sociales tal y como estos ocurren" (Bolsegui y Fuguet Smith, 2006, pp. 208-210).

Coincidimos con los autores mencionados que abordar una investigación desde una perspectiva cualitativa sugiere la consideración de una variedad de componentes de orden filosófico y teorético (interpretativo), de un diseño de investigación apropiado y de métodos adecuados para la recolección y análisis de la información. Tiene el propósito de centrar la atención en los significados que los hombres crean y utilizan para guiar su conducta. De acuerdo con Taylor y Bogdan (1992), la investigación cualitativa constituye un modo de encarar el mundo empírico. A través de este tipo de investigación se producen datos descriptivos en un contexto natural, donde el investigador considera al escenario y a las personas de manera holística, sin pretensión de extraer de ellas la verdad o 
la moralidad, sino su versión detallada, su perspectiva (construcción) del objeto de estudio. Desde este lugar, se afirma que "(...) la investigación cualitativa enfatiza la validez interna, por cuanto sus métodos se ajustan estrechamente a lo que las personas dicen y hacen. La investigación cualitativa proporciona un trato holístico a los fenómenos. La epistemología del investigador cualitativo es existencial y constructivista" (Ibídem, 2006, p. 211).

La comprensión de los fenómenos desde una visión holística requiere considerar contextos variados: temporales y espaciales, históricos, políticos, económicos, culturales, sociales y personales. La mayor parte de los datos que se recogen favorecen una visión personal de la experiencia, lo que facilita al investigador interpretar, reconocer contextos, indagar significados y transmitir un relato basado en la experiencia (Stake, 1998).

Acordamos con Valles (1999, citado en Bolsegui et. al., 2006, p. 209), que "la metodología cualitativa tiene una lógica que sigue un proceso, donde a partir de una experiencia se trata de interpretar el contexto bajo diversos puntos de vista. Su diseño está abierto a la invención (creatividad), la obtención de datos al descubrimiento y el análisis a la interpretación. En la investigación cualitativa el investigador es el principal instrumento de investigación y no los métodos como en la investigación tradicional. Se prioriza su capacidad para relacionarse con los sujetos, su capacidad hermenéutica, su capacidad para facilitar vínculos entre las conexiones y los sujetos" (Taylor y Bogdan, 1986; Sánchez, 2002).

En este sentido la intuición y la creatividad se convierten en elementos claves del proceso de investigación. Bajo esta perspectiva cualitativa la experiencia de investigación que se describe en este trabajo, sólo en su primera etapa -estado del arte-, en el Proyecto de investigación, se contempla también una segunda etapa que estamos comenzando y que consta de tres fases o momentos (que sólo enunciamos a continuación), y que estaremos en condiciones de socializar en otros trabajos, a medida que vayamos avanzando en la investigación con la información obtenida en el campo empírico. Estas fases son: a) Diseño: Se describe el Diseño General de la Investigación, se clarifican los tipos de informantes claves seleccionados, así como la naturaleza del método empleado y las técnicas, como por ejemplo, en el caso de la entrevista cualitativa. Se describen además aspectos referidos al proceso de recolección de información. b) Recolección, construcción y análisis: Se explica que tanto la categorización, como el análisis e interpretación son actividades conjuntas y estrechamente vinculadas entre sí. c) Teorización y construcción del modelo: Se argumenta que la investigación avanza desde una conceptuación preliminar -denominada modelo inicial- que adquiere mayor sentido y profundidad en fases posteriores -modelos intermedios- hasta llegar a una conceptuación final que se presenta como "modelo final" -modelo conceptual-. d) Categorización, análisis e interpretación: "En todo proceso de investigación cualitativa, uno de los aspectos más controvertido, es el análisis. Pareciera que en cuanto a los 
procedimientos de recogida de información existe mayor acuerdo, prolíficas fuentes de consultas, pero en cuanto al análisis aún es insuficiente" (Bolsegui y Fuguet Smith, 2006, p. 210).

Este proceso exige profundizar sobre el material a ser estudiado, desocultar las ideas, construir, deconstruir y volver a integrar articuladamente. Son frecuentes las afirmaciones que aluden al análisis como una perfecta combinación de arte y de procedimientos. Rodríguez y otros (1999) afirman que: "Es preciso mencionar, que a pesar de las dificultades propias del análisis cualitativo, la experiencia al utilizar el método comparativo continuo, es de gran valor. La escritura de los memos, donde se reflejan los "insights" del investigador, ideas relacionadas, propósitos de la investigación, rutas futuras de indagación, nuevos interrogantes; en estrecha combinación con el muestreo teórico contribuyen a superar muchas dificultades. Los memos contienen registros descriptivos y analíticos, fueron utilizados a lo largo de las distintas etapas de la investigación; en correspondencia con los tipos de codificación abierta, axial y selectiva. Los memos facilitan la idea de mantener la investigación vinculada a los datos (grounded) y a la interpretación de los investigadores" (Bolsegui y Fuguet Smith, 2006, p. 227).

\section{La Educación a Distancia. Una alternativa para nuevas prácticas de enseñanza}

El tema de la Educación a Distancia en las Universidades Nacionales ha adquirido una real importancia en los últimos veinte años. La nueva concepción de las Universidades fundada en la democratización de la enseñanza implicó la reconstrucción de una mejor Universidad en calidad de estudio, en producción de conocimientos y en servicios, garantizando el libre acceso, permanencia y egreso de sus estudiantes. Desde este lugar, la modalidad a distancia parece ser una respuesta adecuada para quienes están incorporados al mundo del trabajo o aquellos que habitan en comunidades alejadas de los centros urbanos.

La Educación a Distancia (EAD) es un término genérico, difícil de definir, ya que en él se incluyen las estrategias de enseñanza y aprendizaje que en el mundo se denominan de diferentes formas. Adherimos con Fainholc (1999) en que la EAD "(...) es una forma de educación no convencional que permite llegar a más personas y lugares, utilizando métodos y enfoques innovadores, que puede y debe servir para el logro de la educación permanente". Una estrategia para operacionalizar los principios y fines de la educación permanente y abierta, de manera que cualquier persona, independiente del tiempo y del espacio, pueda convertirse en sujeto protagónico de su aprendizaje (Ramos Martínez, 1985). En este contexto, el proceso de enseñanza y de aprendizaje cobra una importancia fundamental, sobre todo cuando va unido a la comprensión como articuladora de la enseñanza. 
Desde nuestro campo disciplinar, la comprensión se apoya teóricamente en los aportes de Perkins (1999, p. 7) cuando afirma que "comprender es cuestión de ser capaz, y actuar con flexibilidad, a partir de lo que uno sabe. La capacidad de desempeño flexible es la 'comprensión'. (...) La comprensión es abierta y gradual e implica un criterio de desempeño flexible. Como un principio básico, expresa que 'el aprendizaje es una consecuencia del pensamiento'. (...) Las actividades de comprensión requieren pensar. Las imágenes mentalesconocimiento holístico y coherente-favorecen las actividades de comprensión; las actividades de comprensión generan imágenes mentales (reciprocidad). La comprensión de lo particular se inserta en el contexto de la comprensión general".

Destaca cuatro niveles de comprensión:

- Nivel de contenido.

- Nivel de resolución de problemas.

- Nivel epistémico.

- Nivel de investigación.

Se pregunta: ¿Cómo representarnos las cosas para hacerlas comprensibles? Mediante representaciones potentes que proporcionen imágenes mentales que favorezcan la comprensión de los alumnos; se podría recurrir a modelos analógicos, concretos, depurados y construidos.

La pedagogía de la comprensión invita a reorganizar el currículo en torno a temas generadores que den origen y apoyo a diversas actividades de comprensión. Estos temas deben reunir ciertas condiciones: de centralidad, accesibilidad y riqueza. Propone una enseñanza con menos datos, en torno a temas generadores, amplios y fecundos que posibiliten distintos repertorios de comprensión genuina.

Construir y desarrollar un modelo de enseñanza más flexible, incrementa la interacción y facilita la constitución de comunidades virtuales a la vez que promueve la actividad y el aprendizaje colaborativo, otorgando mayor autonomía al alumno.

Internet, en los programas de educación a distancia, puede favorecer a romper la barrera del tiempo y del espacio; facilitar la búsqueda, análisis y producción del conocimiento; generar un nuevo rol para el profesor como propiciador de diferentes desempeños de comprensión auténtica.

La calidad de la educación en este marco se expresa en procesos y resultados genuinos cuando amplía la cobertura de posibilidades educativas. De esta manera, lentamente se va consolidando la tendencia hacia diseños y experiencias en EAD que responden a las necesidades y demandas del estudiantado. Es una opción de enseñanza y de aprendizaje si está acompañada por las Tecnologías de la Información y la Comunicación (TICs) como soportes activos e interactivos que ofrezcan una ventaja diferencial para sus destinatarios, para que puedan avanzar cognitivamente más allá de lo que hubieran 
podido hacer solos. En tanto generen nuevas formas de encuentro, de acceso a la información, al tratamiento de los temas, las formas de plantearlos, como así también, si propenden a una modificación en la estructuración del pensar. Ayudan al estudiante en pensar en sus propios pensamientos.

La difusión de experiencias educativas relacionadas con la enseñanza a distancia tiene un doble beneficio. Por un lado, contribuir al incremento de las capacidades docentes para objetivar la propia acción, reflexionar comprensiva y críticamente sobre ella; y por el otro, la importancia de sistematizarla, comunicar sus hallazgos y dificultades y pensar distintas alternativas de solución a los problemas planteados. "Respecto de las Tecnologías de la Información y la Comunicación (TICs), podemos pensarlas en el marco de la tecnología educativa, entendida como el cuerpo de conocimientos que, basándose en disciplinas científicas referidas a las prácticas de la enseñanza, incorpora todos los medios a su alcance y responde a la consecución de fines en los contextos socio-históricos que le otorgan significación" (Litwin, 1995c).

Para los que venimos trabajando en experiencias en EAD, la práctica de la enseñanza no queda fundamentada únicamente desde lo teórico, sino que implica que cada uno vaya abriendo su propio camino, perfeccionando su campo profesional y personal. En este sentido lo que subyace es que la actuación de los profesores depende notablemente de cómo interpretan el entorno escolar, qué metas persiguen y cómo aprovechan y cualifican la información. Como plantea Edith Litwin (1996), las prácticas de la enseñanza constituyen una totalidad, en la cual se integran las miradas personales de los docentes y la experiencia ganada con el trabajo compartido con otros colegas. Estas prácticas cobran significación y relevancia en el contexto socio-histórico en el que son llevadas a cabo, porque implican una serie de cuestionamientos por parte del docente acerca de cómo enseñar, tanto desde lo moral como desde lo epistemológico. Esto daría cuenta de la buena enseñanza y las buenas prácticas docentes.

Las concepciones sobre el conocimiento docente y la forma en como éste se construye, tienen numerosas relaciones internas con aspectos epistemológicos, políticos, científicos y prácticos, lo que hace que no pueda abordarse su estudio desde un único punto de vista. Popkewitz (1991, p. 110; citado en Litwin, 2000) nos habla de "(...) la importante legitimación científica del conocimiento docente que se habría dado desde los principios del siglo XX en forma, principalmente, de valoración del trabajo relacionado con el currículum y el aprendizaje; al mismo tiempo alerta sobre el peligro de que esto sea utilizado de forma persistente y continuada como estrategia para racionalizar el conocimiento que posee el docente de la práctica pedagógica".

Según el autor al que alude Litwin, sería una muestra de esto que, dada la preocupación administrativa por la educación masiva, no parece un accidente el hecho de que fueran las ciencias experimentales (a partir de las que se propugnaba el análisis empírico y los conocimientos útiles) y el conductismo, los 
enfoques que adhirieron más fuertemente con el diseño instructivo. En definitiva y siguiendo el mismo pensamiento de Litwin, es en el origen de esa legitimación de la que habla Popkewitz, que se pretendía imponer una concepción de que la ciencia traería el "progreso" a las instituciones sociales y económicas y que, en ellas, el conocimiento académico y profesional describiría fenómenos y acontecimientos, pudiendo favorecer los intereses de cualquier grupo social.

En este contexto las ciencias de la educación se preocuparon por contenidos relacionados con la cuestión social: la formación ética y ciudadana, la socialización para el trabajo, los contenidos actitudinales, por el aprendizaje, el estudio de las diferencias de raza, de género, de sexo, de metodologías para el abordaje de la realidad social, etc.

El desarrollo de la educación, y más precisamente de la educación a distancia, cambió su imagen social a partir de la psicología cognitiva que ha sustituido al conductismo como perspectiva relevante en los trabajos y documentos sobre la reforma educativa en nuestro país en las décadas del ' 80 y '90. El investigador cognitivista estudia el pensamiento del profesor sobre las prácticas del aula y este tipo de investigaciones creemos, puede contribuir a identificar determinadas capacidades y habilidades del docente como profesional de la educación.

En un estudio sobre la bibliografía producida por esta corriente intelectual, se pone de manifiesto que los temas de investigación más recurrentes tienden a ofrecer mecanismos de regulación social de las vidas de los profesores. Asimismo, mientras por una parte las investigaciones sobre el pensamiento del profesor aportan elementos de progreso en la concepción de la relación entre teoría y práctica, por otra, la organización de los conocimientos resultantes, introducen nuevas formas de seguimiento y observación del docente en las prácticas cotidianas de su trabajo (áulicas y extraáulicas), en el marco de los estudios etnográficos.

El problema del currículum, profundo y complejo, puede entenderse como un conflicto entre las esperanzas depositadas en la educación y el compromiso de crear, sostener y renovar las condiciones del mundo. Cuando una teoría se aplica a la práctica social, se producen siempre efectos imprevistos y no tenidos en cuenta. De este modo, se plantea la necesidad de pensar modalidades de enseñanza alternativas por fuera de las tradicionales y desde este lugar entender los actos docentes como formas de regulación social, como una selección entre una amplia gama de fenómenos que tiene por objeto circunscribir la interpretación, clasificar, definir lo significativo y trazar los límites que determinarán lo que deberá omitirse: la profesionalización docente debe tener en cuenta estas condiciones del conocimiento como problema filosófico y social.

El conocimiento escolar circula en un entramado de relaciones de poder, de problemas o presiones sociales en relación a los que se ha creado. Esto ha llevado a una educación que contiene pautas de diferenciación, pautas de la 
conversación en el aula y, en consecuencia, las prácticas docentes no responden a normas universales, sino que contienen múltiples planos de significado, interpretación y negociación de los mismos. En síntesis, de singularidades.

Se trataría de entender la educación en general y la enseñanza a distancia en particular, como ámbito que contiene, por una parte, visiones del mundo y, por otra, actuaciones prácticas para la producción y reproducción de ese mundo: de qué es posible hablar, a qué hablantes considerar pertinentes y qué reglas deben ser tomadas en consideración, si partimos por entender que la educación es una práctica social y política compleja, cargada de significado y significantes que amerita ser estudiada y desocultada desde investigaciones educativas como éstas.

\section{La Educación a Distancia en la Universidad Nacional de Rosario}

La Universidad Nacional de Rosario fue creada el 29 de noviembre de 1968 a través de la Ley 17.987. Su estructura fundante fue un desprendimiento de la Universidad Nacional del Litoral, de quien toma sus primeros organismos académicos y administrativos. Actualmente, cuenta con una superficie edilicia de 6,8 ha. donde brinda una oferta académica compuesta de 12 Facultades, 3 Institutos de Enseñanza Media y 1 Centro de Estudios Interdisciplinarios. Brinda una oferta académica compuesta por: 66 títulos de grado, 124 carreras de postgrado, 15 tecnicaturas, 53 títulos intermedios, 26 títulos por articulación con el sistema de Educación Superior no Universitario y 32 postítulos.

A esta oferta académica se le sumó en los últimos años el campus virtual, desde el cual se brindan cursos en la modalidad a distancia empleando como ambiente de enseñanza el soporte Web. Las Unidades académicas que la estructuran son: Facultad de Ciencias Exactas, Ingeniería y Agrimensura (pionera en Educación a Distancia año 1991); Facultad de Ciencia Política y Relaciones Internacionales (EAD comienza en 1996); Facultad de Ciencias Médicas (EAD en la carrera de Enfermería data de 1991); Facultad de Ciencias Bioquímicas y Farmacéuticas; Facultad de Arquitectura, Planeamiento y Diseño; Facultad de Derecho; Facultad de Odontología; Facultad de Ciencias Agrarias; Facultad de Ciencias Veterinarias; Facultad de Ciencias Económicas y Estadística; Facultad de Psicología; Facultad de Humanidades y Artes; Instituto Politécnico Superior "Gral. San Martín"; Escuela Superior de Comercio; Escuela Agrotécnica "Libertador San Martín"; Centro de Estudios Interdisciplinarios.

"El inicio de las gestiones para su creación, está vinculado al reclamo de apertura de la Escuela de Derecho en Rosario. Los primeros años de gobierno se abocaron a la organización de su estructura académica y administrativa, que contaba con las siguientes Facultades, institutos y dependencias: Facultad de Ciencias Médicas (creada en la Universidad Nacional del Litoral en 1919); Facultad de Ciencias 
Exactas, Ingeniería y Arquitectura (creada en la Universidad Nacional del Litoral en 1919); Facultad de Ciencias Económicas (creada en la Universidad Nacional del Litoral en 1919); Facultad de Derecho y Ciencias Políticas (1968); Facultad de Odontología (1968); Facultad de Filosofía (1968); Facultad de Ciencias Agrarias (1966); Los hospitales escuela; Las Escuelas Secundarias que de ella dependen; El Instituto Superior de Música de Rosario; Los organismos directivos, docentes, técnicos y administrativos dependientes del Rectorado, con asiento de la ciudad de Rosario" (Portal www.unr.edu.ar, Consultado el $18 / 02 / 09)$.

El primer Estatuto de la Universidad Nacional de Rosario fue aprobado con fecha del 5 de junio de 1970, por Decreto 2.603/70, en el marco de la Ley 17.245. En octubre de 2001, se creó el campus virtual (PUNTOEDU), que centraliza la educación a distancia de la UNR, se implementó un Programa de capacitación para la administración en el área informática, se concursaron las escuelas medias y se lanzaron e implementaron numerosos acuerdos internacionales, fortaleciéndose sobre todo la participación en el MERCOSUR a través de las actividades de la Asociación de Universidades, Grupo Montevideo.

A través de esta oferta académica del Campus Virtual (PUNTOEDU) se brindan cursos, carreras y postítulos en la modalidad a distancia empleando como ambiente de enseñanza el soporte Web. En este campo, la carrera que se ofrece es la Tecnicatura Universitaria en Administración de Empresas, de 3 años de duración, con posibilidad de optar por distintas especializaciones en el último año de cursado: Servicios, Seguros y Comercio Exterior.

"Cuenta además con una Biblioteca Virtual, que es inaugurada en su Primera etapa en Junio del 2001, durante la gestión del Rector Cont. Ricardo Suárez. Es el proyecto de mayor potencial y envergadura en el tema documental, está integrada por las colecciones de las Unidades de Información, Bibliotecas, Escuelas y Centros documentales de las Facultades de la Universidad Nacional de Rosario. Además posee un Periódico Digital donde se informa acerca de diferentes eventos que dan a conocer en algunas bases de datos científicos disponibles en el portal de la Biblioteca Electrónica del Ministerio de Ciencia, Tecnología e Innovación Productiva de la Nación (MINCYT), a través del cual los usuarios pueden acceder a bibliografía especializada sin costos de suscripción. Dicho acceso debe efectuarse desde terminales conectadas al Servidor de la UNR. La Facultad de Derecho cuenta con una importante AUDIOTECA" (Portal www.unr. edu.ar, Consultado el 18/02/09).

La Biblioteca Virtual brinda posibilidades de acceso a la documentación a través de la Red de Bibliotecas, proporcionando información indistintamente al claustro o Facultad a la que pertenezca, en donde se encuentre, lo que le da 
una fortaleza absoluta ya que cada uno de los Institutos, y sus escuelas están representados en el Catálogo en Línea (OPAC). El propósito es facilitar el acceso de los investigadores, docentes y alumnos de la comunidad universitaria, a la documentación existente en las bibliotecas, centros de información, escuelas, departamentos e institutos que integran la UNR. Un servicio que consideramos medular para los estudios que se realizan con la modalidad a distancia, tanto para carreras de grado como postgrado. Para ello, cuenta con un catálogo de acceso público online que posibilita la recuperación de estos archivos; y con acceso al servidor de la Secretaría de Ciencia y Tecnología -SECyT-, que permite la consulta online de 245 títulos de distintas especialidades.

"El proyecto se inició a mediados de 2001, con fondos de la UNR y del Programa de Financiamiento de Inversiones PROIN. Luego, mediante el plan FOMEC -Fondo de Mejoramiento de la Calidad en Educación Superior-, se incorporaron nuevas tecnologías que permiten informatizar las distintas áreas de la institución, agilizando el tratamiento y la recuperación de información de los usuarios. Ya en 2003, por medio del proyecto SPU, se amplió su parque informático, se modernizaron los espacios para usuarios y se refuncionalizaron sus áreas de trabajo. La biblioteca implementará otro sistema, de registro catalográfico legible por máquina, que la acercará a los estándares internacionales en bibliotecología. Este permite compatibilizar la información para compartirla y, de ese modo, amplía la capacidad de comunicación con sus lectores. Por medio de este software social, los usuarios podrán, por ejemplo, conocer online si un libro de su interés está disponible para ser reservado y con qué número de ejemplares cuenta la biblioteca" (Portal www.unr.edu.ar, Consultado el 15/03/09).

"Nuestra universidad ascendió en el ranking web a nivel mundial, escalando a la posición 1.516 entre 16.000 instituciones universitarias de todo el mundo y se mantiene en el séptimo puesto entre las casas de estudios de la Argentina. El 26 de enero de 2009 el Laboratorio de Cibermetría, perteneciente al CSIC, el mayor centro nacional de investigación de España, publicó su informe semestral que contiene el ranking web de universidades del mundo. El mismo se viene publicando desde 2004, en enero y julio de cada año y está realizado sobre 16.000 instituciones universitarias del mundo, donde 2806 son casas de altos estudios latinoamericanas" (Portal www.unr.edu.ar, Consultado el 15/03/09).

"Nuestra universidad fue ascendiendo desde el segundo semestre de 2007, donde se encontraba en el puesto mundial 1822 al actual 1516 y del puesto 78 al 72 a nivel latinoamericano. Igualmente es para remarcar que todas la universidades argentinas están lejos del Top 
100, solamente tres universidades argentinas están en el TOP 1000, la UBA en el puesto 317, la Universidad Nacional de La Plata en el 528 y la Universidad Nacional de Córdoba en el 904. En el estudio se puede ver la gran diferencia de las universidades de Estados Unidos, con las del resto del mundo. Entre las primeras veinticinco, sólo en el puesto 23 está una universidad de Canadá, las demás son norteamericanas, del puesto veintiséis al cincuenta, se encuentran dos de Canadá, dos de Gran Bretaña (Cambridge, 28 y Oxford, 42), una de Suiza, una de Australia, destacándose como la única latinoamericana a la Universidad Autónoma de México en el puesto 44. La oferta de formación superior de Argentina se ubica entre las mejores de América Latina. Está conformada por 103 instituciones universitarias, que reciben más de 1.500.000 estudiantes: 1.285.000 concurren a instituciones estatales y 250.000 a las privadas. Ubicadas en todas las regiones del país, tanto las universidades como los institutos tienen una oferta académica destinada a estudiantes extranjeros y reciben cerca de 25.000 jóvenes por año. Se pueden cursar carreras de pregrado, de grado y cursos de posgrado. Las carreras de pregrado son cortas, no duran más de cuatro años, y ofrecen títulos técnicos. Las de grado incluyen las licenciaturas, algunos profesorados, medicina, abogacía, las diferentes ingenierías, entre otros. En cuanto a los postgrados la oferta cubre una amplia gama de áreas disciplinares. Por lo general, son arancelados y algunas universidades ofrecen becas para estudiantes extranjeros. (...) El sistema universitario argentino está conformado por: 40 universidades nacionales; 44 universidades privadas; 6 institutos universitarios estatales y 13 institutos universitarios privados; 1 universidad provincial: la Universidad Autónoma de Entre Ríos (UADER) tiene cuatro facultades, con sedes únicamente en esa provincia, y ofrece carreras vinculadas a ciencia y tecnología, ciencias de la gestión, de la salud, entre otras; 1 universidad extranjera: se trata de la Universidad de Bologna, con sede en la Ciudad de Buenos Aires. Su oferta académica incluye maestrías en investigación de mercado, internacionalización del desarrollo local, metodología de la investigación, cursos de alta formación y posgrado sobre la integración europea. Una Universidad Internacional: es la Facultad Latinoamericana de Ciencias Sociales (FLACSO), sede Argentina, donde se dictan tanto en forma presencial como virtual: un doctorado en Ciencias Sociales, trece maestrías y especializaciones, diplomas superiores, cursos y seminarios de posgrado. Esta universidad ofrece becas para extranjeros" (ar.buzzear.net/tag/universidadesnacionales, Consultado el 15/03/09). 


\section{Información relevante obtenida de la búsqueda por la Web}

A continuación, detallamos parte de la información obtenida como resultado de la búsqueda bibliográfica y vía Internet:

Cátedras online que se dictan en las Facultades de: Psicología, Derecho, Ciencias Veterinarias, Ciencias Médicas (Carrera de Medicina). Mencionaremos algunos ejemplos:

1. FACULTAD DE DERECHO: La página de la Facultad de Derecho de la UNR (www.fder.unr.edu.ar) contiene un link al Campus Virtual, dividido en tres secciones:

- Carrera de Abogacía: Cátedras Online (para alumnos de dicha carrera).

- Graduados: Cursos y Carreras de Postgrado Online.

- Comunidades: Acceso a Comunidades Online.

Hemos ingresado al Campus e indagado en la sección 1, y encontramos que se puede acceder a dichas cátedras de dos formas:

- LIBRE: Permite visitar la página de la cátedra y obtener material disponible, de forma pasiva (sólo lectura).

- $\quad$ USUARIO: Requiere de un nombre de usuario y clave que provee el docente de la cátedra. Participación activa, ya que permite agregar archivos e interactuar con otros miembros.

Exploramos las distintas cátedras a través del acceso LIBRE, y encontramos como cátedra pionera en implementar la modalidad a distancia a Derecho Procesal I (Cát. A); de hecho en la presentación a la materia se hace referencia al surgimiento de dicha implementación (año 2005). Dispone de un índice con los temas a trabajar, y de 52 archivos entre ellos bibliografía, programa, calificaciones, TP, lecturas sugeridas.

Una cátedra que presenta una experiencia "innovadora" es Derecho Civil II (Cát. B). Con el nombre "Clase Taller": Discriminación y Derecho Privado, los docentes le presentan a sus alumnos la "primer experiencia de educación intranet en la Comisión 11 de Obligaciones". Además les proporcionan una serie de "tips" que los estudiantes deben tener en cuenta para realizar las consignas que se les propone semanalmente. Las dudas que se les presenten pueden ser "evacuadas" en las clases presenciales y las actividades están coordinadas y seguidas vía intranet. (Consultado en el Campus, noviembre de 2008).

\section{FACULTAD DE CIENCIAS MÉDICAS:}

Carrera de Medicina: En la página de la Facultad (www.fcm.unr.edu.ar) encontramos el Campus Virtual donde deberían aparecer cátedras online. Se solicita nombre de usuario y clave. También aparece un campo llamado "Módulo de Inclusión Universitaria" (MIU), destinado a los Ingresantes 2008, donde se les da la bienvenida a los mismos, incluyéndose información sobre el cursado de la carrera y el transcurso por la vida universitaria. Además contiene un "Anexo" con una serie de documentos sobre Derechos Humanos y Derecho a la Salud. 
Independientemente de la página de la Facultad, encontramos sitios webs de distintas cátedras, a saber:

- www.clinica-unr.org Primera Cátedra de Clínica Médica y Carrera de Especialización en Clínica Médica. Este sitio contiene información sobre la cátedra, el cuerpo docente, la investigación, la Educación a Distancia (se dictan cursos online). Posee tres divisiones: Grado, Medicato y Posgrado.

- En "Grado", aparece como primer link "Enseñanza de la Clínica Médica”, a cargo del Titular Dr. Alcides Greca; también se incluyen una serie de monografías realizadas por alumnos y seleccionadas por el cuerpo docente, y la distinción entre los dos programas vigentes en la Facultad: Plan 1998 y Plan 2001, con el correspondiente cronograma de clases, cursado, exámenes, noticias, material recomendado.

- La página contiene, además, referencias a libros, links a revistas digitales, imágenes e información sobre distintas patologías, seminarios, información a la comunidad, entre otras cosas.

- $\quad$ www.farmacologiaunr.com Esta página corresponde a la página de Farmacología, y encontramos información sobre el cuerpo docente, material de estudio, actividades, multimedia, investigación, entre otras.

- $\quad$ www.hepagastro.org Si bien esta página aparece titulada como Servicio de Gastroenterología y Hepatología del Hospital Provincial Centenario, contiene un link donde se explicita la Formación de Grado. Pueden descargarse clases teóricas, se incluye un cronograma para los planes 1998 y 2001, noticias y fechas de exámenes.

Carrera de Enfermería: Se implementa en dos ciclos: 1) Formación de Enfermeros. 2) Licenciatura en Enfermería. Desde 1991, comenzó a dictarse con la Modalidad a Distancia, cubriendo la demanda de formación de varias regiones del país, a través de Sedes. Hoy atraviesa una crisis institucional.

3. FACULTAD DE PSICOLOGÍA: La página Web de la Facultad (www. fpsico.unr.edu.ar) contiene una "Plataforma Virtual", donde los alumnos pueden acceder, a través de un tutorial, al contenido de las distintas materias de grado. Allí aparece el programa de la materia, los contenidos, actividades, los docentes y sus producciones, futuros eventos, novedades, etc. Todos los alumnos pueden acceder a ella, estén activos o no.

4. FACULTAD DE CIENCIAS VETERINARIAS: Al igual que Derecho y Medicina, esta Facultad contiene, en su página web (www.fveter.unr.edu.ar), un Campus Virtual. Para acceder al mismo se necesita nombre de usuario y contraseña, proporcionado por las cátedras que han optado por introducir esta modalidad de enseñanza. Similar a la experiencia de Derecho, los alumnos pueden obtener información sobre sus notas, materiales de la cátedra, información sobre el cursado, noticias, entre otras. Esta información la obtuvimos a 
través de una charla con una alumna que está cursando el segundo año de la carrera, ya que mediante la web no pudimos acceder. Esta experiencia comenzó a implementarse en el año 2008, siendo la cátedra "pionera" Anatomía.

Tanto la Facultad de Ingeniería como la Escuela de Comunicación Social son las de mayor trayectoria en relación a la implementación de proyectos e investigación en torno a la modalidad de enseñanza a distancia, no así en el dictado de carreras de grado, como lo es la Licenciatura en Enfermería de la Facultad de Ciencias Médicas.

5. FACULTAD DE CIENCIAS EXACTAS, INGENIERÍA Y AGRIMENSURA: Considerada institución pionera en la modalidad a distancia en carreras de grado. "Hacia fines de 1990 se propuso la incorporación de dicha modalidad como una alternativa que permite dar una respuesta válida a la demanda social de educación en áreas científico-tecnológicas, siendo las principales áreas de interés: el acceso a carreras de grado; la actualización y el perfeccionamiento docente en los distintos niveles del sistema educativo; la capacitación científicotecnológica; la formación y actualización de posgrado" (http://www.fceia.unr. edu.ar/inicio, Consultado el 09/04/08).

La capacitación en la modalidad a distancia del personal de la Facultad está coordinada académicamente por la Dra. Marta MENA, reconocida especialista en EAD de la Universidad de Buenos Aires. El llamado Período Introductorio para ingresantes a las carreras de Ingeniería (años 1992 a 1994) constituyó el inicio de las actividades a distancia desarrolladas institucionalmente. A partir de 1994 la Facultad cuenta con un espacio institucional específico para la promoción y el desarrollo de actividades en el área de la EAD: el Departamento Educación a Distancia, dependiente de la Escuela de Postgrado y Educación Continua. "La misma se propone la promoción y el desarrollo de actividades tendientes a posibilitar la no presencialidad para: la actualización y perfeccionamiento docente en los distintos niveles del sistema educativo; educación continua en Ingeniería; formación de postgrado; capacitación a la comunidad; el diseño de estrategias que posibiliten crear ambientes de aprendizaje más flexibles en el contexto de la enseñanza presencial". Esta información la encontramos en la página Web del Departamento de Educación a Distancia de dicha Facultad, donde se expresa que "(...) la calidad de sus propuestas, es una preocupación permanente. Dicho Departamento, junto al área de desarrollo de tecnología educativa de la Facultad está incorporando en sus cursos, el empleo de diferentes medios. Se busca crear en ellos ambientes de aprendizajes adecuados, mediante entornos virtuales que promuevan el aprendizaje colaborativo y el establecimiento de la comunicación con fines didácticos y administrativos. Al respecto, y a partir de una primera experiencia realizada en la asignatura Física IV en 1998, hoy distintas cátedras han iniciado el camino de la integración de tecnologías de la información como estrategia de comunicación y recurso para las enseñanzas 
en el contexto de Proyectos específicos de innovación en las enseñanzas de Ingeniería" (http://www.fceia.unr.edu.ar/inicio, Consultado el 09/04/08).

Creemos pertinente mencionar algunos trabajos, publicaciones, ponencias, seminarios, artículos, que dan cuenta de dicha trayectoria. Entre las publicaciones y ámbitos científicos de actuación de los docentes de la Facultad de Ciencias Exactas, Ingeniería y Agrimensura, encontramos en su página Web lo siguiente:

- 'Influence of text based instruction on teachers' conceptual change in Science: a research within the distance course "Energy. Its Rational Use" - 1999, S. Marchisio - M. Massa - P. Sánchez - E. Llonch, Publicado en CD-ROM e INTERNET por el Comité Organizador del 19th International Council of Distance Education World Conference, Viena, Austria.

- $\quad$ "La creación de valor en una organización educativa: el caso de un Departamento Universitario proveedor de cursos a distancia en la Argentina" - 1999, S. Marchisio, trabajo inédito desarrollado en el marco de estudios de Doctorado, Escuela Técnica Superior de Ingenieros Industriales, UNED, Madrid, España".

- "The contribution of tutorial action to the design and development of the evaluation of the distance course: "Probability, Statistics and Quality Control" - 1999, M. Anido - R. Katz - S. Marchisio - Publicado en CD-ROM e INTERNET por el Comité Organizador del 19th International Council of Distance Education World Conference, Viena, Austria.

- "The new learning environments in the University Education: an experience of integration of the technologies of information in a traditional Physics course in Argentina" - 1999, O. Von Pamel - S. Marchisio - Publicado en CD-ROM e INTERNET por el Comité Organizador del 19th International Council of Distance Education World Conference, Viena, Austria.

- "El diseño del material escrito para resignificar contenidos y prácticas de enseñanza sobre ENERGÍA" - 1998, S. Marchisio - M. Massa; Memorias en formato electrónico (diskette) del III Seminario Internacional de Educación a Distancia de RUEDA (Red Universitaria de Educación a Distancia de Argentina) - Córdoba, Argentina.

- $\quad$ "Probabilidad, Estadística y Control de Calidad. una asignatura con modalidad tutorial en el postgrado de Ingeniería Gerencial" - 1998, R. Katz - S. Marchisio; Memorias en formato electrónico (diskette) del III Seminario Internacional de Educación a Distancia de RUEDA (Red Universitaria de Educación a Distancia Argentina), Córdoba, Argentina.

- "La educación a distancia como alternativa en la formación continua del ingeniero: una experiencia piloto en el Postgrado de Ingeniería Gerencial" 1998, R. Katz - S. Marchisio - M. Pujol, aceptado para publicación en Anales del $2^{\circ}$ Congreso Argentino de Enseñanza de la Ingeniería, organizado por la Universidad de San Juan, Argentina. 
- "Acerca del ámbito de actuación didáctica del profesor de Ciencias" 1998 - S. Marchisio - M. Massa, Memorias del $4^{a}$ Simposio de Investigadores en Enseñanza de la Física - SIEF4- Universidad Nacional de La Plata - La Plata, Argentina.

- Aportes al diseño de un sistema de calidad de un Departamento de Educación a Distancia en una institución de educación superior de enseñanza presencial" - 1998, S. Marchisio, trabajo inédito desarrollado en el marco de estudios de Doctorado, Escuela Técnica Superior de Ingenieros Industriales, UNED, Madrid, España.

- "Validation of distance education programs within a traditional face to face institution in Argentina", 1997, S. Marchisio, Publicado en CD-ROM e INTERNET por el Comité Organizador del 18th International Council of Distance Education World Conference, Pennsylvania State University, USA.

- "Making up of action projects in a class situation as means for testing teachers' training in Natural Sciences in Argentina" - 1997, M. Massa - S. Marchisio - M. Yanitelli - H. D’Amico - P. Sánchez - S. Cabanellas - E. Llonch; Publicado en CD-ROM e INTERNET por el Comité Organizador del 18th International Council of Distance Education World Conference, Pennsylvania State University, USA.

- "La actuación docente de los profesores de ciencias, ¿un ambiente de aprendizaje en educación a distancia?” - 1997, S. Marchisio - M. Massa - P. Sánchez; Publicación en formato electrónico "Memorias del VI Encuentro Internacional de Educación Abierta y a Distancia”, Universidad de Guadalajara, en el marco de la Feria Internacional del Libro de Guadalajara, México.

- LLa escuela y los problemas derivados del consumo de energía: una experiencia en la formación docente a distancia" - 1997, S. Marchisio - M. Massa - S. Cabanellas - P. Sánchez - M. Yanitelli - H. D’Amico - E. Llonch; $7^{\circ}$ Encuentro de la Asociación Iberoamericana de Educación Superior a Distancia (AIESAD) - Río de Janeiro, Brasil.

- "Distance Education as an Alternative In Teaching Training In Relationship to Natural Sciences in Argentina" - 1996, M. Massa, S. Marchisio - M. Yanitelli - H. D'Amico - P. Sánchez - S. Cabanellas - E. Llonch; Proceedings de ICDED 96 - Conferencia Internacional de Educación a Distancia en Rusia, Moscú.

- "Distance Education: an Educational Alternative in Continuous Engineering Education" - 1995, S. Marchisio - M.E. Piantanida, Proceedings de la 6th. World Conference on Continuous Engineering Education (6th. WCCEE), International Association for Continuing Engineering Education (IACEE) y UNESCO, San Pablo y Río de Janeiro, Brasil. 
- "Desarrollo del tema Energía y su Uso Racional en un curso a distancia para docentes" - 1995, M. Massa - S. Marchisio - P. Sánchez - H. D’Amico - S. Cabanellas - M. Yanitelli - E. Llonch; Publicación de ASADES 94: 17 Reunión de trabajo de la Asociación Argentina de Energia Solar y 3 Encuentro Nacional de la International Association for Solar Energy Education (IASEE), organizado por IFIR, CONICET, UNR; Rosario, Argentina.

- "Análisis numérico matricial con herramientas computacionales: un curso a distancia en el ámbito de la educación continua" - 1995, M. Anido de López - S. Marchisio - M. Medina - H. Rubio Scola - M. Yanitelli, Anales del "Congreso Internacional de Informática Educativa", (GIAIT - GIAGSE - Proyecto SAE) - Facultad Regional Santa Fe - UTN, Santa Fe, Argentina.

- "Educación a Distancia: una alternativa que trasciende nuestras aulas" 1995, S. Marchisio; "75 AÑOS" - Publicación de la Facultad de Ciencias Exactas, Ingeniería y Agrimensura de la UNR, Argentina.

- "Evaluación del Período Introductorio para Ingresantes 1994 a Distancia" 1994, S. Marchisio, Publicación interna de la Facultad de Ciencias Exactas, Ingeniería y Agrimensura (U.N.R.), Argentina.

- “Curso Introductorio para Ingresantes 1993" - 1993, D. Asteggiano - M. Guzmán - S.Marchisio; Publicación interna de la Facultad de Ciencias Exactas, Ingeniería y Agrimensura (U.N.R.), Argentina.

- "Proyecto de Organización del Área Educación a Distancia" - 1992, S. Marchisio - M.Guzmán - M.E. Piantanida; Publicación interna de la Facultad de Ciencias Exactas, Ingeniería y Agrimensura (U.N.R.), Argentina.

- "Propuesta de formación docente a distancia con un enfoque constructivista" - 1992, M. Massa - S. Marchisio - P. Sánchez - H. D’Amico - M. Yanitelli - E. Llonch - S. Cabanellas; Revista Brasileira de Ensino de Física, Vol. 14, $N^{\circ}$ 3. (http://www.fceia.unr.edu.ar/inicio, Consultado el 12/04/2008). NALES:

6. FACULTAD DE CIENCIA POLÍTICA Y RELACIONES INTERNACIO-

Carrera de Comunicación Social: Materias de primer año (años 1997 a 2000). A partir del año 1997 esta Escuela realizó experiencias de Educación a Distancia en las cátedras de Antropología, Taller de Redacción I, Historia Argentina y Latinoamericana y en el curso de orientación para ingresantes. Desde el año 2001 se viene implementado un nuevo Plan de Estudios, donde esta modalidad prácticamente ha desaparecido. Sólo algunas cátedras han continuado implementando e investigando acerca de esta modalidad de enseñanza y aprendizaje, mediatizadas por las TICs. En 2004, se pone en práctica la construcción de una experiencia educativa generada a partir de la utilización de software social, que intenta construir nuevos escenarios de diálogo, descentralizar el conocimiento, reconfigurar las relaciones de jerarquía docente/ alumno, y que propende al desarrollo de cogniciones distribuida, a través de 
la inclusión digital y la apropiación de espacios privados, no configurados para propuestas educativas.

- La Propuesta digital Dialógica: potrero de experimentación en comunicación y educación, funciona bajo la dirección del Prof. Fernando Irigaray como Coordinador del Área de Desarrollo Multimedia (Rosario, Pcia. de Santa Fe Argentina, 21 Marzo de 2004). A través del Centro de Comunicación y Educación de dicha escuela, se trabaja mediante una Tutoría en Internet (weblog) con los Ingresantes 2008. Entre las cátedras que implementan weblogs con sus alumnos, se encuentran: Redacción I, Pensamiento Sociopolítico II, Institución y Sociedad, Comunicación Audiovisual (Prof. Irigaray), Seminario de Integración y Producción (www. fcpoli.unr.edu.ar, s.f.).

En relación a las publicaciones en Revistas científico-académicas, seminarios, simposios de los docentes de la Escuela de Comunicación Social, citamos lo siguiente:

1) En el Anuario de Comunicación Social y Trama de la Comunicación (UNR) encontramos:

1.1- "Comunicación Social: De la fantasía a la realidad". Pallavicini, M. y otros, Escuela de Comunicación Social Facultad de Ciencia Política y Relaciones Internacionales. 1996. UNR, Rosario. Argentina.

1.2- "Prácticas alternativas de la Enseñanza". Pallavicini, M. En Anuario del Dto. De Comunicación Social, 1998/99, Vol. 4, Facultad de Ciencia Política y Relaciones Internacionales, UNR, pp. 61-66, 1996. Rosario. Argentina.

1.3- "Curso de Orientación para Ingresantes a Comunicación Social: Estrategias para reducir la deserción". Pallavicini, M. y otros. En Anuario del Dto. De Comunicación Social. Año 1998/99, Vol. 4, pp. 291-311. Universidad Nacional de Rosario, Rosario. Argentina.

1.4- "Aportes de Antropología a la Educación a Distancia. Algunas reflexiones". Taborda, M. y Copertari, S. En Anuario del Departamento de Comunicación Social, Año 1998/00, Vol. 5, pp. 9-20. Facultad de Ciencia Política y Relaciones Internacionales, UNR. Rosario. Argentina.

1.5- "Tutorías electrónicas en Redacción I". Perelli, L. En Anuario "La Trama de la Comunicación" Escuela de Comunicación Social Facultad de Ciencia Política y Relaciones Internacionales, UNR, Vol. 8, pp. 21-27. 2002/2003, Rosario. Argentina.

1-6- "Tecnología y Educación. Evaluación de una experiencia de Educación a Distancia como Proyecto Tecnológico Educativo". Copertari, S. En Anuario de Comunicación Social. Facultad de Ciencia Política 
y Relaciones Internacionales, UNR, 2003/2004, Vol. 9, pp. 123-131, Rosario. Argentina.

2) En la Revista de la Escuela de Ciencias de la Educación (UNR) hemos localizado:

- Artículo presentado en la Revista de la Escuela de Ciencias de la Educación. Año 3. № 2. Laborde Editor. Rosario. "Investigación y Prácticas Docentes. Procesos Metacognitivos en la Enseñanza a Distancia”. Copertari, S. La autora comenta algunas reflexiones que surgieron de una experiencia de Educación a Distancia realizada en la Facultad de Ciencia Política y Relaciones Internacionales (UNR), en los años 1999-2000, y enfatiza en la importancia que adquiere la investigación educativa del docente sobre su propia práctica en el marco del Proyecto de Investigación Pol94/06.

3) En Ponencias Presentadas a Congresos, Seminarios, Jornadas, Encuentros, etc.:

- "Enseñanza de Redacción a Distancia I", Margarit, Ana y otra. Escuela de Comunicación Social. Facultad de Ciencia Política y Relaciones Internacionales, UNR, Ponencia presentada en la 3ra. Reunión Regional de América Latina y el Caribe, ICDE, Sao Pablo. Brasil. Publicado por Internet (Argentina, 2000).

- Trabajo presentado por la Escuela de Ciencias de la Comunicación en el Congreso de Educación a Distancia en el $3^{\text {er }}$ Milenio. UNESCO, AUGM. "La experiencia del Curso para ingresantes a la Carrera de Comunicación Social en 1997 con modalidad a Distancia”. Pallavicini, M. y otros (1998).

- "Educación a Distancia: una práctica alternativa en el Nivel Universitario con Ingresantes a Comunicación Social, en la Universidad Nacional de Rosario". Taborda, M., Copertari, S y otros (Argentina). Ponencia presentada en el IV Taller Internacional de Educación a Distancia (La Habana, 17 al 21 de mayo de 1999), Cuba.

- "Educación a Distancia: un nuevo desafío en Tiempos de Globalización. Construyendo ambientes de aprendizaje en Educación a Distancia". Taborda, M. y Copertari, S. Ponencia presentada al 3ra Reunión Regional de América Latina y el Caribe. ICDE (Sao Pablo del 15 al 17 de agosto de 2000), Brasil.

\section{Algunas reflexiones a modo de cierre}

Luego del recorrido realizado en esta fase de la investigación sobre el estado actual de los conocimientos sobre el tema, podemos delinear sólo algunas reflexiones como producción de un primer acercamiento con las experiencias. La problemática central de la investigación se irá desentramando a medida que 
podamos avanzar con el trabajo en el campo empírico, a partir del contacto con informantes-clave.

Estamos convencidos que la educación a distancia no pretende suplantar la enseñanza tradicional presencial, sino que puede pensarse como una modalidad alternativa que la enriquece y complementa. Coincidimos con Marta Mena (2007) cuando dice que para que se implementen buenas prácticas y buenas enseñanzas en la modalidad, habrá que indagar en profundidad los programas de apoyo académico. Las buenas prácticas resultarán adecuadas al relacionar directamente el contexto y la propuesta de intervención educativa analizadas desde el método comparativo constante de las prácticas de enseñanza. Esto es, cuando pueda hablarse de una práctica contextualizada -con cierta coherencia entre teoría y práctica- y cuando se logren cumplir los objetivos propuestos.

Otro factor determinante es que la propuesta de enseñanza esté encarada integralmente a través de materiales didácticos impresos, virtuales, interactivos y multimediales, acompañados por una interacción permanente de todos los participantes. Si bien Internet es un medio válido y potente como recurso educativo, no es un soporte neutral, inocente y productivo que sólo requiere de una conexión apropiada para convertirse en un integrante más de la situación de enseñanza en un espacio educativo. No alcanza con poner aulas en red, si están disociadas de esas redes las cuestiones curriculares, didácticas y cognitivas que le dan sentido a su inclusión en el sistema educativo (Mena, 2007).

Deberemos corroborar, en las experiencias investigadas, si se plantean un conjunto de estrategias -fundamentalmente cognitivas y metacognitivas- con dispositivos especialmente diseñados, como apoyo al aprendizaje del estudiante, como por ejemplo: consultoría virtual, foros, libros electrónicos, servicios en línea, bibliotecas, entre tantos otros. Tendremos que analizar, si la/s tecnología/s seleccionada/s se integra/n al programa a distancia y aporta/n a la obtención del objetivo propuesto, respetando las características del mismo y de los destinatarios. Y si todos estos procesos, están monitoreados permanentemente por una investigación evaluativa, que priorice los procesos cualitativos por sobre los cuantitativos y que nos permita obtener información relevante acerca de su funcionamiento. Estas cuestiones podremos profundizarlas a partir de la información que arroje el trabajo empírico y a la par de este proceso de análisis crítico y reflexivo, poder pensar en una política en relación a los docentes, que se traduzca en acciones que favorezcan la formación de capital humano para la gestión del conocimiento (Trottini, 2007) y, en educación a distancia como propuestas alternativas de la educación tradicional exclusivamente nutrida en la presencialidad.

La Educación a Distancia y los nuevos entornos virtuales de enseñanza y aprendizaje están ganando terreno cada vez más; con la incorporación del aula virtual surgen nuevos espacios que requieren de una formación permanente y continua de los educadores que se ocupan fundamentalmente de la Educación 
Superior Universitaria. El docente que se apropie de nuevas herramientas de trabajo, podrá descubrir una innovadora forma de comunicarse con sus alumnos, de enriquecer su práctica o bien de difundir, publicar, exponer su currículum, sus producciones, saberes, opiniones, investigaciones, etc. sin dejar de lado el mero hecho de hacerse conocer a través de la red o interactuar con comunidades virtuales afines con su interés. "(...) Una producción no implica siempre el desarrollo de un material didáctico, puede haber una producción donde se expongan contenidos o donde se brinde información" (larmusch, 2005, p. 87).

Nuestra intención con esta investigación, es realizar un aporte a la Universidad Nacional de Rosario, a las Unidades Académicas involucradas y a la Educación Superior en general, a fin de rescatar prácticas de enseñanza innovadoras de la modalidad tradicional puramente presencial en las carreras de grado, como resultantes de un análisis comparativo en el marco de las configuraciones didácticas de "buenas enseñanzas", que tiene que ver con el proceso de democratización de la enseñanza y la nueva agenda didáctica de la Educación a distancia en particular, en el contexto de la educación virtual para el Siglo XXI (2) y (3).

\section{Notas Bibliográficas}

(1) El Equipo de investigación está integrado por Susana Copertari (Directora), Silvia Morelli (Co-Directora); Ana María Trottini (Asesora); Elsie Laurino; Gaspar Aita; Juan Emilio Contesti (Integrantes); Yanina Fantasía; Ma. Laura Ruiz y Natalia Salomoni (Becarias-Tesistas).

(2) En parte esta producción fue socializada en una Ponencia presentada ante el III Congreso Nacional y II Encuentro Internacional de Estudios Comparados en Educación: Copertari, S., Morelli, S., Trottini, A.M.; Fantasía, Y., Contesti, J.E., Aita, G., Stara, N. "Análisis de experiencias sobre prácticas de enseñanza en Educación a Distancia en la Universidad Nacional de Rosario". Organizó SAECE, Buenos Aires, 25 al 27 de Junio de 2009. Expositora: Fantasía, Y.

(3) Por tratarse de un recorrido sobre el "Estado de la cuestión" de la problemática que se plantea, parte del análisis que se presenta en la Introducción, el cuerpo de desarrollo y las reflexiones finales de este trabajo, se encuentran en una publicación de Copertari, S., Morelli, S., Trottini, A.M.; Fantasía, Y., Contesti, J.E., Aita, G., Stara, N.: "Análisis de experiencias sobre prácticas de enseñanza en Educación a Distancia en la Universidad Nacional de Rosario", en Revista Digital Cognición (FLEAD) № 18 (2009). Fundación Latinoamericana para la Educación a Distancia. En el presente trabajo se incorpora un desarrollo sobre el abordaje metodológico, que se materializará en la segunda etapa del proceso investigativo sobre el campo empírico, a partir del mes de Octubre de 2009. 


\section{Referencias Bibliográficas}

- Achilli, E. (1998) La Práctica docente: una interpretación desde los saberes del maestro. Cuadernos de Formación Docente. Rosario, UNR Editora.

- Baker, L. (1994). "Metacognición, lectura y educación científica”, en Inc, C. y otra (Comp.) Una didáctica de las ciencias, procesos y aplicaciones (pp. 2223). Buenos Aires, Aique.

- Bolsegui, M. y Fuguet Smith, A. (2006) "Construcción de un modelo conceptual a través de la investigación cualitativa", en Revista Universitaria de Investigación Sapiens, Vol. 7, № 1, Junio, pp. 206-232. ISSN 1317-5815. Caracas, Venezuela.

- Bromme, R. (1988) "Conocimientos Profesionales de los Profesores", en Revista de Enseñanza de las Ciencias № 6, pp. 19-29. University Biefefeld.

- Camilloni, A. (1996) Corrientes didácticas contemporáneas. Buenos Aires, Paidós.

- Clark, B. (1983) El sistema de educación superior: una visión comparativa de la organización académica. Nueva Imagen. Azcapotzcalco, México, Universidad Autónoma Metropolitana.

- Clark, C. (1999) "Procesos de pensamiento de los docentes", en Wittrock, M.C. (Comp.) La investigación en la enseñanza III. Barcelona, Paidós.

- Coria, A (s.f.) Palabras e imágenes a distancia. Material del Seminario de Formación Docente (1998). Rosario, Facultad Ciencia Política y RRII. UNR.

- Copertari, S., Morelli, S., Trottini, A.M., Laurino, E., Aita, G., Constesti, J.E., Stara, N., Fantasía, Y., Ruiz, M.L. y Salomón, N. (2009) "Experiencias de prácticas de enseñanza en Educación a Distancia en carreras de grado de la Universidad Nacional de Rosario". Ponencia presentada en el Congreso de Pedagogía 2009 en el Simposio 19. Educación Superior; Actividad: Tecnologías de la Información y la Comunicación IV; Ubicación 1; Sala 11; Día: 2009-01-28; Hora 4:10 pm. (La Habana-Cuba). Expositora: Psicopedagoga Bilicich, C.

- Copertari, S (2006) "Tecnología y educación a distancia desde la complejidad", en La Trama de la Comunicación. Dpto. de Comunicación Social. Facultad de Ciencia Política y RRII. Vol. 11 (Año XI), pp. 357-370. UNR Editora. ISSN Nº 1668-5628. Rosario.

- Doval, L. y Peña, S. (2005) "Educación y Nuevas Tecnologías. Módulo 1. Fundamentos y Perspectivas de los recursos pedagógicos y de las TICs en educación", en Capacitación Directiva en Gestión de Recursos Tecnológicos en La Escuela. Universidad Nacional de Catamarca. CAPACYT. Formación Docente SRL. Buenos Aires.

- Fainholc, B. (1999) La interactividad en la Educación a Distancia. Buenos Aires, Paidós.

- $\quad$ Forni, F. (1993) Métodos cualitativos II. Buenos Aires, Eudeba.

- García Garrido, M. (1976) La Universidad Nacional de Educación a Distancia. Madrid, CEAC. 
- Gardner, H. (1997) “Las inteligencias múltiples", en Zona Educativa. Ciencias de la Educación $N^{\circ} 18$. Año 2. Buenos Aires, Ministerio de Cultura y Educación de la Nación.

- Hudder, I. (1994) "The interpretation of Documents and Material Culture", en Denzin, N.K. y Lincoln, Y. (Eds.) Handbook of Qualitative Research. Thousand Darks CA, Sage Publications.

- $\quad$ larmusch, S. y Rainoldi, A. (2005) "Módulo Tecnologías para la Comunicación Educativa”, en Gestión Pedagógica de las Tics. Universidad Nacional de Catamarca. Buenos Aires, CAPACYT/Formación Docente.

- Lago Martinez y otros (Coord.) (2003) En torno de las metodologías: abordajes cualitativos y cuantitativos. Buenos Aires, Proa XXI.

- Litwin, E. y otra (1990) La educación a distancia. Deseos y realidades. Buenos Aires, UBA/OEA.

- Litwin, E. y otra (1991) La educación a distancia. Deseos y realidades. Buenos Aires, Miño y Dávila.

- Litwin, E. y otros (1994) Educación a Distancia en los '90. Desarrollos, problemas y perspectivas. Facultad de Filosofía y Letras (UBA) y Programa UBA XXI. Buenos Aires.

- $\quad$ Litwin, E. (1995a) "Educación a Distancia: una modalidad en desarrollo", en Revista Versiones $\mathrm{N}^{\circ} 3-4$, pp. 15-18. Buenos Aires.

- Litwin, E. (1995b) "La evaluación como una explicación ecológica de la actividad en el aula", en Evaluación. Aportes para la Capacitación N 1, pp. 45-65. Ediciones Novedades Educativas $N^{\circ} 57$. Buenos Aires.

- Litwin, E. (Comp.) (1995c) Tecnología Educativa. Política, historias, propuestas. Buenos Aires, Paidós.

- $\quad$ Litwin, E. (1996) "El campo de la Didáctica: la búsqueda de una nueva agenda", en Camilloni, A. (Comp.) Corrientes Didácticas Contemporáneas. Buenos Aires, Paidós.

- Litwin, E. (1997) "Las configuraciones didácticas en el marco de una Nueva Agenda Didáctica", en Revista del IICE, pp. 42-51. Buenos Aires.

- Litwin, E. (Comp.) (2000) La educación a distancia. Temas para el debate de una nueva agenda educativa. Buenos Aires, Amorrortu.

- Lugo, M.T. (1999) Capacitación a Distancia: acercar la lejanía. Herramientas para el desarrollo de programas a distancia. Buenos Aires, Magisterio Río de la Plata.

- Mena, M. (1996) "La evolución de la educación a distancia" [en línea] http:// weblog.educ.ar/educacion-tics/cuerpoentrevista.php?idEntrev=50 (Consultado el 04/03/2009).

- Mena, M. (2002) "Calidad educativa en los entornos virtuales" [en línea] http:// www.mujeresdeempresa.com/capacitacion/capacitacion020702.shtml (Consultado el 04/03/2009). 
- Mena, M. (2004). "La educación combinará el aula con la virtualidad" [en línea] http://www.elearningworkshops.com/modules.php?name=News\&file=article\&s id=290 (Consultado el 04/03/2009).

- Morin, E. (1995) Introducción al Pensamiento Complejo. Barcelona, Gedisa.

- Pérez Lindo, A., Trottini, A.M. y otros (2005) Gestión del Conocimiento. Un nuevo enfoque aplicable a las organizaciones y la universidad. Buenos Aires, Norma.

- Perkins, D. (1995) La escuela inteligente. Barcelona, Gedisa.

- Popkewitz, T. (1991) A political sociology of educational reform: Power/ knowledge in teaching, teacher education and research. New York, Teachers College Press.

- Gimeno Sacristán, J. y Pérez Gómez, A. (1992) Comprender y transformar la enseñanza. Madrid, Morata.

- Sanjurjo, L. (2002) La formación práctica de los docentes. Rosario, Homo Sapiens.

- Stone Wiske, M. (Comp.) (1999) La enseñanza para la comprensión. Vinculación entre la investigación y la práctica. Barcelona, Paidós.

- Taylor, S.J. y Bodgam, R. (1992) Introducción a los métodos cualitativos de investigación. Buenos Aires, Paidós.

- Trottini, A.M. (2007) La especificidad de la Gestión Universitaria. Gobierno y Administración de su capital humano. Rosario, UNR Editora, Colección Académica.

- Vasilachis De Gialdino, I. (1993) Métodos cualitativos II. La práctica de la investigación. Buenos Aires, Eudeba.

- Zabalza Beraza, M. (1998) "Pensamiento del profesor y desarrollo didáctico", en Revista Didáctica. Salamanca. España. 